\title{
Surgical Outcomes of Pediatric Patients with Asymptomatic Tethered Cord Syndrome
}

\author{
Toshitaka Seki ${ }^{1}$, Kazutoshi Hida ${ }^{2}$, Shunsuke Yano ${ }^{2}$, Kiyohiro Houkin ${ }^{1}$ \\ ${ }^{1}$ Department of Neurosurgery, Hokkaido University Graduate School of Medicine, Sapporo, Japan \\ ${ }^{2}$ Department of Neurosurgery, Sapporo Azabu Neurosurgical Hospital, Sapporo, Japan
}

\section{Study Design: A retrospective cohort study.}

Purpose: To examine the validity of prophylactic surgery for children with tethered cord syndrome (TCS).

Overview of Literature: Prophylactic surgery for pediatric patients with TCS remains controversial.

Methods: We retrospectively analyzed the surgical outcomes of 14 children (nine boys and five girls) with asymptomatic TCS who were surgically treated at Hokkaido University Hospital between 1989 and 2015.

Results: The median age at the time of initial surgery for asymptomatic TCS was 28.6 months (range, 0-66 months). The median final follow-up period was 142 months (range, 7-232 months). Of the 14 children with asymptomatic TCS, 12 had lumbosacral lipoma and two had meningocele. According to the classification of spinal lipoma, two children had dorsal type, four had caudal type, two had transitional type, and four had filar type. There were no children with lipomyelomeningocele. All children were free of neurological symptoms until 94 months after the initial surgery. Subsequently, one child exhibited delayed neurological deficits and underwent a second surgery because of motor and sensory disturbances; slight sensory disturbance was noted at the final follow-up examination. Another child later showed bowel and bladder dysfunction. However, a second surgery was not performed for this child because his motor and sensory functions were normal; hence, we chose to avoid nerve injury in the case of dissecting adhesion.

Conclusions: All 14 children with asymptomatic TCS were free of neurological symptoms until 94 months after the initial surgery. However, two children exhibited delayed neurological deficits at 94 months and 177 months. We believe that prophylactic surgery for asymptomatic TCS is effective for a certain period. However, because the natural history of TCS is poorly understood, strict follow-up after surgery is necessary.

Keywords: Child; Lipoma; Spinal dysraphism; Prophylactic surgery; Outcome

\section{Introduction}

Tethered cord syndrome (TCS) is the clinical manifestation of an abnormal stretch of the spinal cord and is presumed to cause mechanical injury, a compromised blood supply, and altered spinal cord metabolism. TCS is associated with neurological deficits in the lower limbs, leg numbness, lower back pain, foot deformities, and bowel and bladder disturbances [1,2]. It is unlikely that symptoms will sufficiently improve if surgery is performed after the onset of neurological symptoms associated with TCS. Hence, prophylactic surgery for asymptomatic TCS is advocated and performed. In the present study, the surgical outcomes of children with asymptomatic TCS were

Received Sep 3, 2017; Revised Oct 9, 2017; Accepted Oct 10, 2017

Corresponding author: Toshitaka Seki

Department of Neurosurgery, Hokkaido University Graduate School of Medicine, N-15, W-7, Kita-ku, Sapporo, 0608638, Hokkaido, Japan

Tel: +81-11-706-5987, Fax: +81-11-708-7737, E-mail: toseki1@hotmail.com 
retrospectively analyzed, and the validity of prophylactic surgery for asymptomatic TCS was examined.

\section{Materials and Methods}

Between 1989 and 2015, 14 children with asymptomatic TCS underwent surgical treatment at Hokkaido University Hospital. Patients with presacral lipomas, other spinal dysraphisms (such as split cord malformations), or severe neuro-orthopedic deformities (such as clubfoot or worsening scoliosis) were excluded from this study. We explained the surgical procedure and obtained prior in-

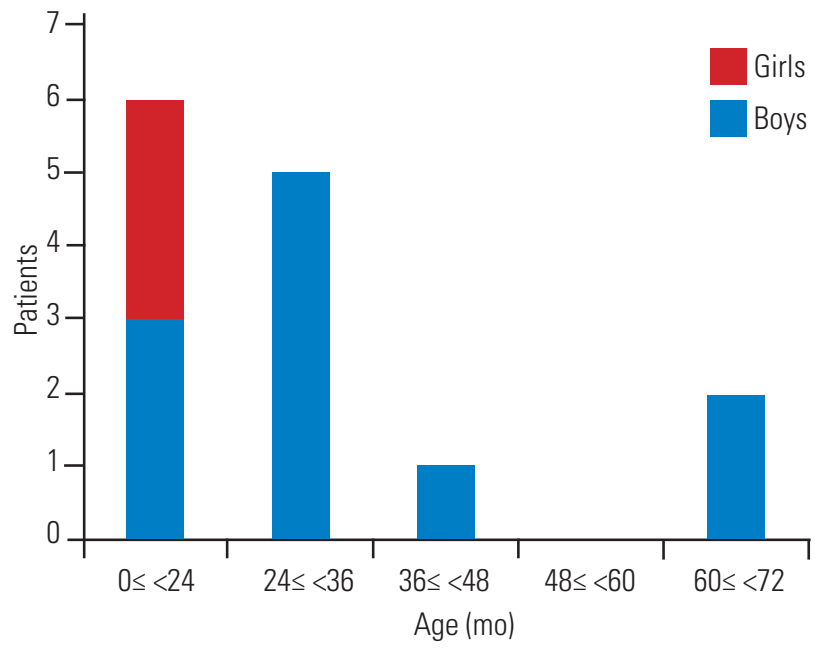

Fig. 1. Age (in months) of patients with asymptomatic tethered cord syndrome at the initial surgery. formed consent from all patients. The surgical outcomes of these children were retrospectively reviewed.

\section{Results}

All 14 children (nine boys and five girls) with asymptomatic TCS underwent prophylactic surgery to release the tethered conus. The median duration of follow-up was 142 months (range, 7-232 months).

The median age at the time of the initial surgery was 28.5 months (range, 0-66 months) (Fig. 1). Six children underwent the initial surgery when aged $<24$ months, which was the most common age period. Subsequently,

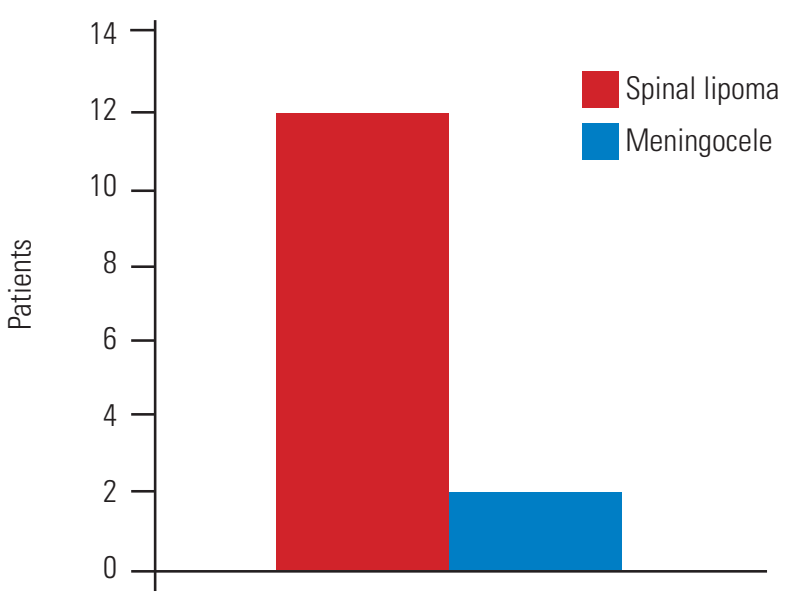

Fig. 2. Lumbosacral spine lesion in patients with asymptomatic tethered cord syndrome. Twelve patients had lumbosacral lipoma and two had meningocele.
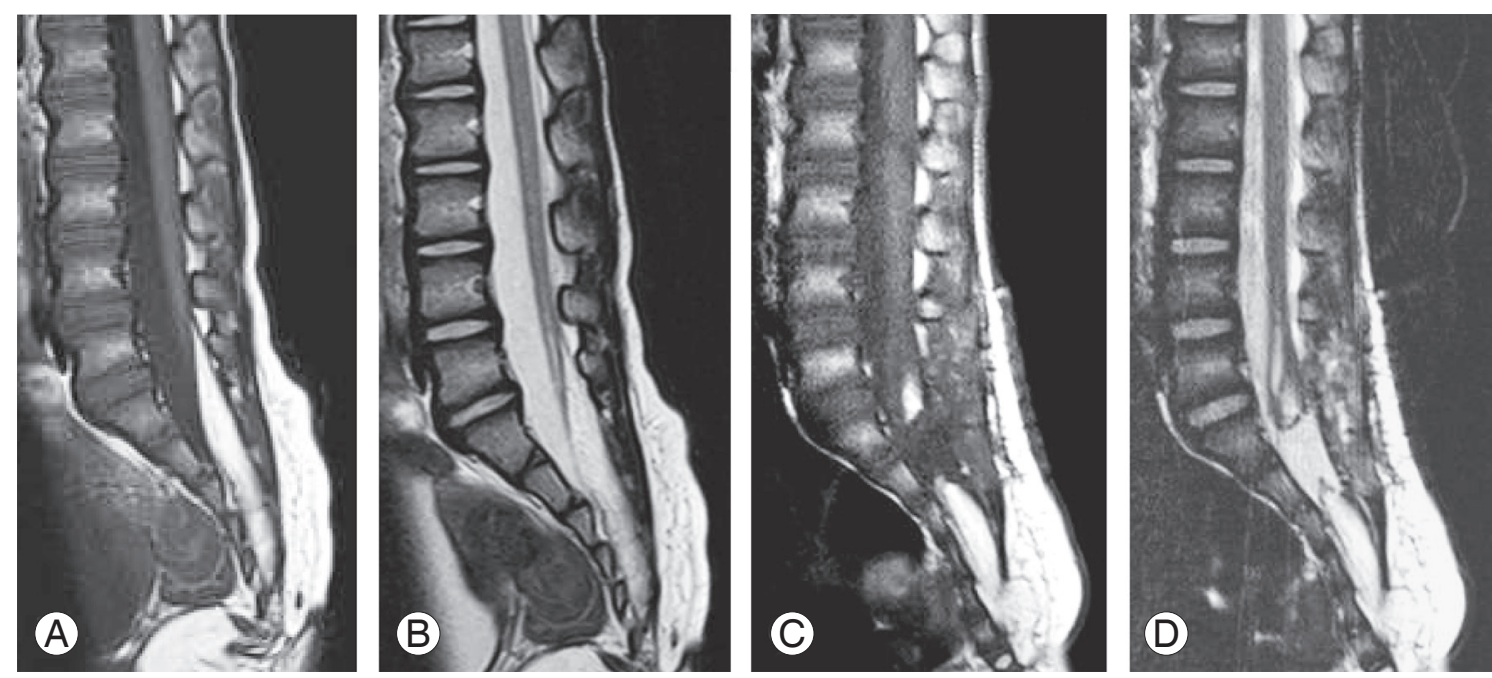

Fig. 3. Preoperative sagittal T1-weighted (A) and T2-weighted (B) MRI showing a transitional lipoma in a 2-year-old boy. Postoperative sagittal T1-weighted (C) and T2-weighted (D) MRI performed 1 month after surgery showing partial removal of the lipoma and forward movement of the spinal cord. MRI, magnetic resonance imaging. 
five children underwent prophylactic surgery when aged between 24 and 36 months. Two children underwent surgery when aged $>60$ months.

Of the 14 children with asymptomatic TCS, 12 had lumbosacral lipoma and two had lumbosacral meningocele (Fig. 2). The classification of spinal lipoma was dorsal type in two children, caudal type in four, transitional type in two, and filar type in four. There were no children with lipomyelomeningocele. The level of conus medullaris determined via magnetic resonance imaging (MRI) was L2-3 in one child, L3-4 in two, L4-5 in one, L5 in two, S1 in three, S2 in two, and S3 in three. Bipolar stimulation and continuous electromyography were performed for all patients during surgery. Although intraoperative evoked potential monitoring became available only in the last part of the present series, we did not use it because it may trigger epileptic seizures. Treatment consisted of surgical untethering of the spinal cord, partial resection of the spinal lipoma (in case of lipoma), and creation of subarachnoid space, where possible. Next, the dural sac was constructed by direct dural repair using GORE-TEX threads (W. L. Gore and Associates Inc., Newark, DE, USA). The extradural space was sealed using fibrin glue (Beriplast P Combi-Set Tissue Adhesion; CSL Behring, King of Prussia, PA, USA). Afterward, the superficial layers were reconstructed in the usual manner. Fig. 3 shows preoperative and postoperative MRIs for a representative patient.

All children were free of neurological symptoms until 94 months after surgery (Fig. 4). Subsequently, two boys exhibited delayed neurological deficits: one had caudal type spinal lipoma at 94 months, and the other had dorsal type spinal lipoma at 177 months. One child underwent a second surgery because of deterioration of neurological function exhibiting as motor and sensory disturbances. Slight sensory disturbance was noted at the final followup examination. The other child showed bowel and bladder dysfunctions; however, a second surgery was not performed because his motor and sensory functions were normal, and we thought it best to avoid nerve injury in the case of dissecting adhesion (Table 1).

\section{Discussion}

It is currently unclear whether prophylactic surgery for children with asymptomatic TCS can prevent the development of future neurological deficits. Regarding the natural course of asymptomatic TCS, conversion to symptomatic TCS is reported at a rate of $3 \%-4 \%$ per year [3-5]; hence, it seems natural to consider preventive surgery. However, several studies investigating the success of early preventive surgery have reported conflicting outcomes. Kulkarni et al. [3] performed conservative therapy in 53 patients with lipomas of the spinal cord conus. During the 9-year follow-up period, there was no significant difference in the appearance of neurological symptoms between the conservatively treated and early surgery groups. However, the expression rate of delayed neurological deficits was lower in the conservative therapy group $33 \%$ in the conservative therapy group versus $46 \%$ in the early surgery group). Wykes et al. [5] performed conservative treatment for 56 patients with asymptomatic lipomas of the spinal

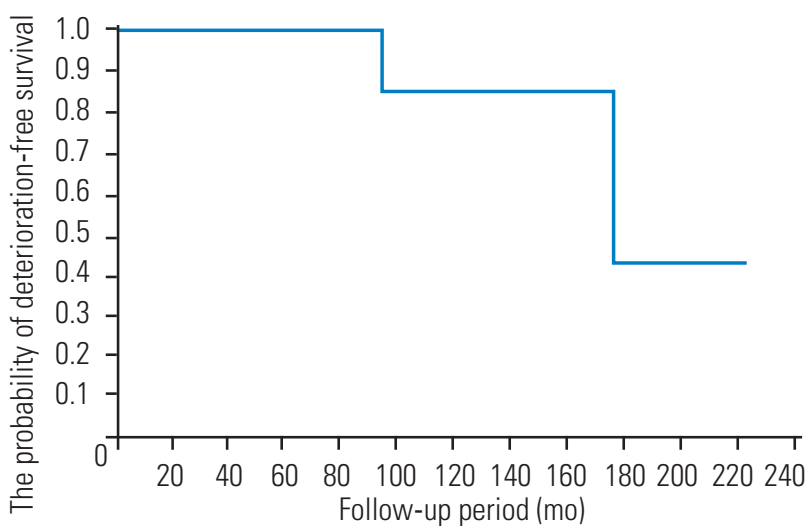

Fig. 4. Survival curve showing the deterioration-free survival of patients who underwent prophylactic surgery.

Table 1. Patients with delayed neurological deficits

\begin{tabular}{lccccccc}
$\begin{array}{c}\text { Sex/age at initial } \\
\text { surgery (mo) }\end{array}$ & $\begin{array}{c}\text { Type of } \\
\text { spinal lipoma }\end{array}$ & $\begin{array}{c}\text { Delayed neurological } \\
\text { deficits (mo) }\end{array}$ & Symptom & $\begin{array}{c}\text { Age at 2nd } \\
\text { surgery (mo) }\end{array}$ & $\begin{array}{c}\text { Final } \\
\text { F/U }\end{array}$ & $\begin{array}{c}\text { Motor or sensory } \\
\text { disorder at F/U B at F/U }\end{array}$ \\
M/16 & Caudal & 94 & BB & None & 195 & None & Continue \\
M/7 & Dorsal & 177 & $\begin{array}{c}\text { Motor and sensory } \\
\text { deficits }\end{array}$ & 184 & 232 & Sensory & None \\
\hline
\end{tabular}

M, male; BB, bowel and bladder dysfunction; F/U, follow-up. 
cord conus with $71 \%$ of patients being asymptomatic during the mean follow-up period of 5.9 years. Furthermore, the hazard ratio of becoming symptomatic was $40 \%$ in 10 years. Both groups had a negative opinion of prophylactic surgery for asymptomatic TCS. Regarding delayed neurological deficits after prophylactic surgery for asymptomatic TCS, Arai et al. [6] reported that three (8.3\%) out of 36 cases exhibited delayed neuropathy 3-6 years after surgery. In these patients, no lesions, such as a re-tethered cord or syringomyelia, were detected on MRI. Hence, the authors considered dysplasia of the lower spinal cord to be involved with the onset of symptoms. In contrast, La Marca et al. [7] reported that 28 patients with asymptomatic filum terminale lipoma who underwent prophylactic surgery exhibited no delayed neurological deficits (mean follow-up period, 3.4 years). However, of 71 patients, nine $(12.7 \%)$ with asymptomatic spinal cord conus lipomas exhibited delayed neurological deficits after prophylactic surgery. Talamonti et al. [4] performed prophylactic surgery (mean follow-up period, 9.7 years) or conservative therapy (mean follow-up period, 10.4 years) for 56 patients with asymptomatic spinal conus lipomas. During the follow-up period, delayed neurological deficits were observed in $9.7 \%$ of patients who underwent prophylactic surgery and in $29.1 \%$ of those who underwent conservative therapy. Although there was no statistically significant difference between both groups, the results suggested that prophylactic surgery is useful for asymptomatic spinal cord conus lipomas ( $p=0.067$ ). In our study, the results of prophylactic surgery for 14 children with asymptomatic TCS revealed that all children remained asymptomatic until 94 months after the initial surgery. However, two children (14.3\%) exhibited delayed motor and sensory disturbances or bowel and bladder dysfunctions due to a re-tethered cord. Of these children, one underwent reuntethering surgery.

Although the small patient population is a limitation of our study, the results seem acceptable given the annual incidence of delayed neurological deficits is 3.7\%-4.0\% as reported by Kulkarni et al. [3] and Wykes et al. [5] (annual deterioration rate in our study, $1.8 \%$ ). Kanev and Bierbrauer [8] retrospectively analyzed children with lipomeningocele and showed that the incidence of neurological symptoms increased with increasing age. Regarding the surgical timing for asymptomatic TCS, Seki et al. [9] reviewed 31 pediatric patients with TCS and reported that patients with asymptomatic TCS underwent surgery significantly earlier than patients with symptomatic TCS $(p=0.045)$. In addition, Hoffman et al. [10] examined 97 patients with lipomyelomeningocele and found that $62.5 \%$ of patients aged $>6$ months who underwent prophylactic surgery remained asymptomatic, whereas $70.7 \%$ of those aged $>6$ months who underwent prophylactic surgery became symptomatic. Taken together, these reports suggested that the age of the patient during prophylactic surgery for TCS is a risk factor for the onset of neurological symptoms. Furthermore, the age of the patient should be carefully considered during prophylactic surgery for asymptomatic TCS.

\section{Conclusions}

We performed prophylactic surgery for 14 children with asymptomatic TCS. All children remained asymptomatic until 94 months after the initial surgery. However, two patients later exhibited delayed neurological deficits. We believe that prophylactic surgery for asymptomatic TCS is effective for a certain period; however, because the natural history of asymptomatic TCS remains unknown, strict follow-up after surgery is necessary.

\section{Conflict of Interest}

No potential conflict of interest relevant to this article was reported.

\section{References}

1. Izci Y, Kural C. Composite type of split cord malformation: rare and difficult to explain. Pediatr Neurosurg 2011;47:461.

2. Selcuki M, Unlu A, Ugur HC, Soygur T, Arikan N, Selcuki D. Patients with urinary incontinence often benefit from surgical detethering of tight filum terminale. Childs Nerv Syst 2000;16:150-4.

3. Kulkarni AV, Pierre-Kahn A, Zerah M. Conservative management of asymptomatic spinal lipomas of the conus. Neurosurgery 2004;54:868-73.

4. Talamonti G, D’Aliberti G, Nichelatti M, Debernardi A, Picano M, Redaelli T. Asymptomatic lipomas of the medullary conus: surgical treatment versus conservative management. J Neurosurg Pediatr 2014;14:245-54.

5. Wykes V, Desai D, Thompson DN. Asymptomatic 
lumbosacral lipomas: a natural history study. Childs Nerv Syst 2012;28:1731-9.

6. Arai H, Sato K, Okuda O, et al. Diagnosis and treatment of lumbosacral lipomas. Jpn J Neurosurg 1998;7:484-90.

7. La Marca F, Grant JA, Tomita T, McLone DG. Spinal lipomas in children: outcome of 270 procedures. Pediatr Neurosurg 1997;26:8-16.

8. Kanev PM, Bierbrauer KS. Reflections on the natural history of lipomyelomeningocele. Pediatr Neurosurg 1995;22:137-40.
9. Seki T, Hida K, Yano S, et al. Surgical outcome of children and adolescents with tethered cord syndrome. Asian Spine J 2016;10:940-4.

10. Hoffman HJ, Taecholarn C, Hendrick EB, Humphreys RP. Management of lipomyelomeningoceles: experience at the Hospital for Sick Children, Toronto. J Neurosurg 1985;62:1-8. 